\title{
A Cross-Platform Application Framework for HTML5-based e-Services
}

\author{
Dan Johansson and Karl Andersson \\ Department of Computer Science, Electrical and Space Engineering \\ Luleå University of Technology \\ SE-931 87, Skellefteå, Sweden \\ Email: \{dan.johansson, karl.andersson\} @ltu.se
}

\begin{abstract}
The e-service area is faced with unique challenges and opportunities as a new mobile IT-landscape emerges. Technical challenges such as heterogeneous devices and networks, scalability and ability to deliver e-services anytime, anywhere to the users must be met with the careful design of functional application platforms and means of distributing e-services in an efficient manner. In this paper, we present a novel cross-platform application framework for HTML5-based e-services. We analyse the framework, applying our NIMO model for development and deployment of e-Services, proving advantages concerning abstraction, availability and accessibility, as well as deployment and functionality.
\end{abstract}

Keywords-Mobile e-Services; HTML5; Application Framework; e-Service Model

\section{INTRODUCTION}

Recent statistics [1] show that the number of mobile broadband subscriptions has exceeded one billion worldwide. The number of new subscriptions grow annually at a rate of $40 \%$, and with the good coverage of $3 \mathrm{G}$ networks and the advent of new and faster standards, user demands concerning e-services increases.

The public sector faces many challenges when it comes to providing e-services to the citizens. Simplifying daily life through personally adapted services, available anywhere, anytime are important targets for municipalities and governmental organizations.

When it comes to e-services, there are many stakeholders. The users of the services often form a very heterogeneous group, with differences in e.g. age, gender, authority levels, and service level expectancy. Other stakeholders include the service owners, the service administrators or operators, the service developers, the service integrators/deployers, the service maintainers, the service regulators, the third party service providers, and service innovators. [2]

First generation e-services included one-way delivery of static information over simple web pages, while second generation e-services open up for limited interaction through simple web-based forms. Municipalities and government agencies today typically offer third generation trusted e-services allowing for secure and rich interaction with their clients and citizens. Moreover, fourth generation e-services are just around the corner embracing the world of smartphones and tablets al- lowing citizens to use their favourite gadgets while interacting with municipalities or government agencies, making e-services truly mobile. As a consequence, the rich feature set of the most advanced smartphones and tablets allow for very advanced and easy-to-use location-based e-services, multimedia enhanced eservices, and multimodal e-services.

One option for municipalities and government agencies would, of course, be to deploy native apps for the mobile device platforms/operating systems their clients and citizens typically use. That would in many cases mean developing apps in parallel for iOS AppStore, Google Play, and Microsoft's Windows Phone marketplace. On the contrary we argue developing cross-platform HTML5-based e-services is much a better alternative in most cases.

In this paper, we present a novel cross-platform application framework for HTML5 e-services. We analyse the framework, applying our NIMO model for development and deployment of e-Services, from many aspects stressing the framework as a good example of future e-service platforms.

The rest of this paper follows a disposition where we present related work (Section II), followed by a description of our case: the "My Skellefteå" framework (Section III). We then analyze the case applying a model for development and deployment of e-Services (Section IV). We conclude the paper through summarizing our work and discussing future work (Section V).

\section{RELATED WORK}

Olsson and Rönnbäck [3] discussed collaboration with various stakeholders in different scenarios analyzing four different development projects pointing out pros and cons in the various approaches studied. Important lessons learnt include were identified in the three alternatives of user involvement, in the studied development projects, namely development for the user, development with the user, and development by the user. The authors conclude that existing business models of software suppliers do not foster innovation in developing new e-solutions and that collaborative approaches provide better end results.

User involvement and citizen driven development is however not uncomplicated. Gidlund [4] studied the process of citizen driven development of public e-services and concluded 
that there is an overreliance and an uncritical acceptance of citizen driven development of e-services on a policy level. Gillig and Sailer [5] focused on user involvement in the e-Services innovation process. A framework for analysing existing involvement activities and for generation of new forms of user involvement was developed. Six dimensions were included in the framework, which takes into account internal and external factors affecting the companies innovation activities. Grimstad and Myrseth [6] pointed out the importance of information governance and systematic work with metadata and semantics as a basis for cross-sector e-Services in public administration. They recommended to increase the understanding of metadata strategies, having top management to pay attention to these issues, and having a new or existing public agency to operate a national metadata service.

Looking at the design of e-services, Hellman [7] presented seven guidelines adding temporal accessibility to e-services, being overview and general information, targeted and relevant information, safety and trust, supper to multi-channel platform and "family resemblance", logical process and progression, storage and retrieval of information, and timeline. The author points out that it must be easy for the user to find, learn, recognize, and recall e-Services. Finally, the author claims existing design principles support the implementation of the proposed principles increasing the temporal accessibility of e-Services. Guidelines for the design of mobile e-services (defined as e-services offering full service mobility, increased functionality due to terminal and user mobility, cross-platform functionality, and support for offline usage) were presented in [8]. Mobile e-services were proven qualitatively different from traditional e-services, with a dedicated set of guidelines being design for: application and service accessibility, individualization, location utilization, platform independence, service mobility, two-way communication, and usefulness. Böcker et al. [9] described a method for identifying enablers for future e-Services. The method identifies usability problems with future interaction technologies and maps them into e-Services. The authors claim using the proposed method would allow stakeholders to spot potential difficulties in the design of user interfaces and preventing usability issues.

Ntshinga [10] defined intelligent e-Services by supplementing the definition for e-Services by adding an intelligent capability for the purpose of effective and efficient choreography of processes. The reason for doing this was to enable composition of intelligent e-Services in a manner encouraging the interoperability of a range of services pertaining to various autonomous virtual enterprises.

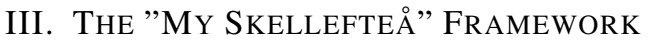

"My Skellefteå"1 [11] is a public framework for e-Services targeted towards the just over 70.000 citizens within the Swedish municipality of Skellefteå. The key component is an application acting as a "container" for different services. The user can decide for herself which services to be included in the

\footnotetext{
${ }^{1}$ In the original Swedish framework the name is "Mitt Skellefteå"
}

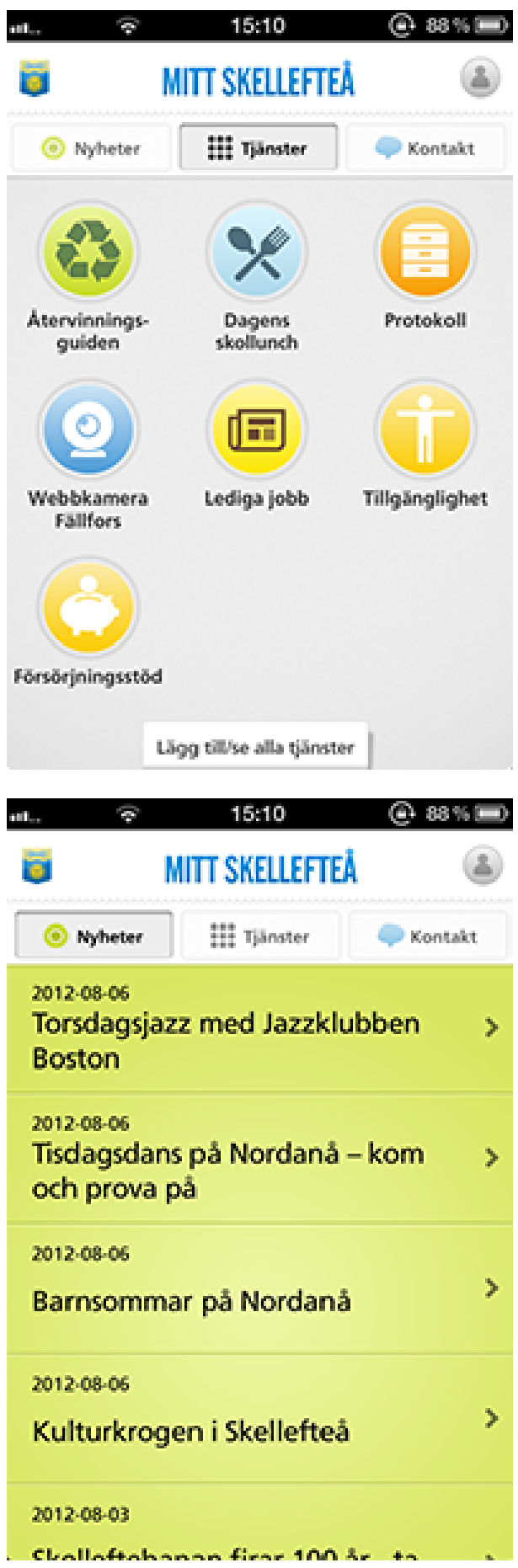

Fig. 1. Container application screenshots 


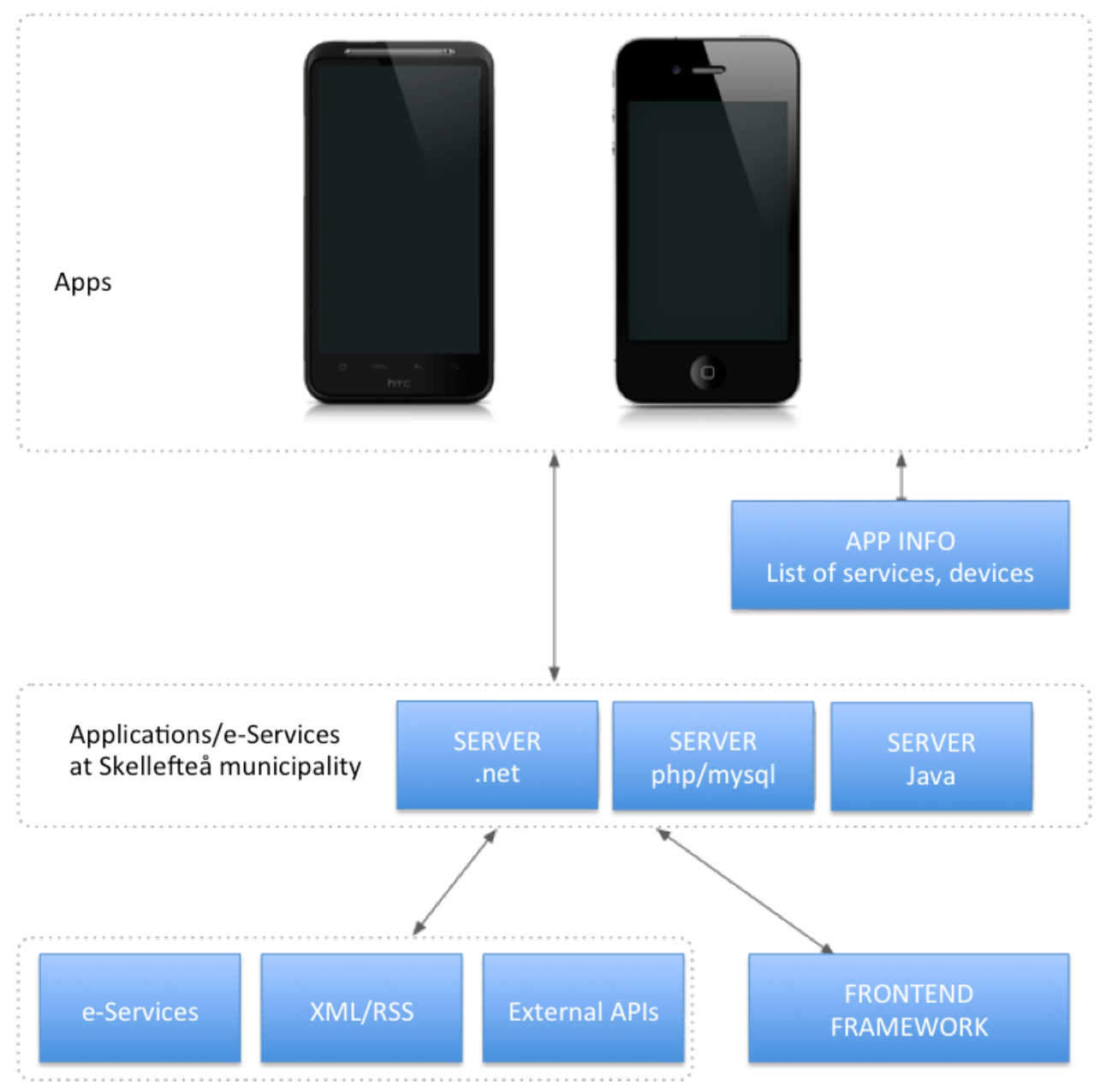

Fig. 2. The "My Skellefteå" framework

application among all available e-services at Skellefteå municipality, e.g. recycling guides, opening hours for libraries, etc. The application can also be used for contacting officials and consume news concerning the municipality. (Screenshots are depicted in Figure 1.)

The container application exists in both iOS and Android versions, downloadable from AppStore and GooglePlay respectively, free of charge. The services embedded within the container application are designed using the emerging HTML5 standard, being supported by most modern browsers. All service development is therefore cross-platform, cross-browser supportive.

Developing e-services for the "My Skellefteå" framework is not restricted to developers within the organization - everyone can become a developer! A developer only has to register her Device ID to the municipality, then she is free to start designing applications to publish within the container application. Any webkit-browser (e.g. Safari or Chrome) can be used as a testbed, and the same goes for the iOS simulator Xcode. HTML5 and CSS3 templates are provided to facilitate development, while guidelines for graphics, writing and general tone helps developers to stay consistent to the general look and feel of the services deployed within the container application. Through this openness, the novel framework allows stakeholders to take on several roles, being e.g. both users, developers and integrators of services.

Besides the container app, the technical architecture consists of applications and services hosted by Skellefteå municipality, running on servers supporting different execution environments. The services can communicate with other services through APIs, both internal and external. Finally, there is also a catalogue service, listing the different services available. This catalogue also contains information about devices. The technical architecture is shown in Figure 2.

\section{ANALYSIS}

We apply the NIMO (Nordic Interaction and Mobility Research Platform) model [12] for development and deployment of mobile e-Services, analyzing the Application Framework for HTML5-based e-Services deployed in the municipality. The NIMO architecture consists of a four layer model, where the device layer encompasses the hardware used for harbouring 
network interfaces and applications, but also the tactile communication between the e-service and the user, often using the browser as a tool for interaction. The network layer abstracts the delivery of data and contains networks like Personal Area Networks (PAN), Wireless Local Area Networks (WLAN), Wireless Metropolitan Area Networks (WMAN) and Wireless Wide Area Networks (WWAN). An important part of the model is the service support layer. This layer contains entities supporting the actual e-Services, still not being e-Services in themselves. E.g. can asset management and location management be used to integrate the end-user's current location with municipality assets to enhance an e-Service with user mobility data. Community management is also handled by this layer, providing social media integration with Twitter, Instagram, Facebook, etc. Identity management is also taken care of at the service support layer, e.g. allowing electronic IDs to be used for authentication of individual users. The NIMO model is depicted in Figure 3.

The "My Skellefteå" framework corresponds well to the NIMO architecture model. The e-Service layer contains the actual e-Services, divided into nineteen areas where some are clusters of services concerning e.g. high school or camping within the municipality, while others are standalone eServices, e.g. water meter readings or car parking. Protocols for communication with the other layers in the NIMO model are also provided, mainly implemented through XML-based data delivery mechanisms.

The service support layer consists of the server architecture, supporting .NET, PHP/MySQL and Java respectively. Internal as well as external APIs provide additional functionality at this layer. Among the most important internal APIs are the FF.Application and FF.Page, equivalents to jQuery, focusing on information retrieval; FF.GoogleAnalytics, for statistics; and FF.Modal for alerts and user dialogue. Also, there are no limitations for the incorporation of external APIs. This makes it possible to fully integrate APIs like GeoLocation, allowing an application to query the browser about the current location of the user (or rather the device); Web Workers, to run JavaScripts in the background in parallel, creating multiple threads; Notification and Vibration, for push functionality; and Web Sockets for full duplex communication between the server and the client, just to name a few [13]. The current version of the framework has built-in support for push notification and camera access, improving the embedded functionality. With every update of the framework, dependency of external APIs decreases.

The network layer is not a part of the "My Skellefteå" framework. The layer design allows for any means of network interfaces to be used, as long as they are based on TCP/IP. In the case described in this paper, the most commonly used networks are IEEE 802.11 (WiFi) combined with IEEE 802.3 (Ethernet) mainly delivered though the municipality WMAN, and various public cellular networks.

The application is designed entirely using the HTML5 emerging standard. This opens up for a wide range of devices to be used within the device layer. Smartphones and tablets from different vendors, running either Android or iOS, can function as tools for the user to consume the e-Services provided through the framework.

Summarizing our analysis, designing cross-platform application frameworks for HTML5-based e-Services using the NIMO model has proven advantageously in many ways, most notably concerning:

- Abstraction. The layered model provides developers with a synoptic view of the framework and, more importantly, the means of easy service development and deployment. Developers get to work with already available APIs and UX/GUI guidelines and templates, enabling them to focus fully on functionality and the actual service composition. Communication and service support is easily available through communication with the responsible module, be it authentication or location queries.

- Availability and Accessibility. Provided with the functionality embedded within the device and network layers, mobile e-Services can be used through any available network interface, on every kind of device running a browser. The HTML5/CSS3 integration allows for easy design of adaptable graphical user interfaces in cases were internal templates are found insufficient.

- Deployment. As the application functions as a container for interfaces connecting to the e-Services, developers can continuously work with deployment without being locked in or dependent of external platforms like Appstore and Google Play, as in line with the NIMO model. The time span between development and deployment is kept short.

- Functionality. Integration of both internal and external APIs in the service support layer opens up for a wide range of features to implement with the service and give developers the possibility to enhance mobile e-Services beyond a mere translation of a traditional web-based eService to a mobile device. The heterogeneous nature of devices and users is taken into account, much as a result of the openness to various APIs.

\section{CONCLUSION}

In this paper, we presented a novel cross-platform application framework for HTML5 e-services. We analysed the framework, applying our NIMO model for development and deployment of e-Services, proving advantages concerning abstraction, availability and accessibility, as well as deployment and functionality. We conclude that a framework designed around the NIMO model is a strong way to answer up to modern challenges within the e-service are, such as users with heterogeneous devices, different vendors providing different networks, the ever increasing need for scalability and the ability to deliver e-services anytime, anywhere.

As for future work, we will evaluate the "My Skellefteå" framework regarding user acceptance and also in terms of the ability to support heterogeneity, scalability, and deliverability, thus further validating our recent findings. The 


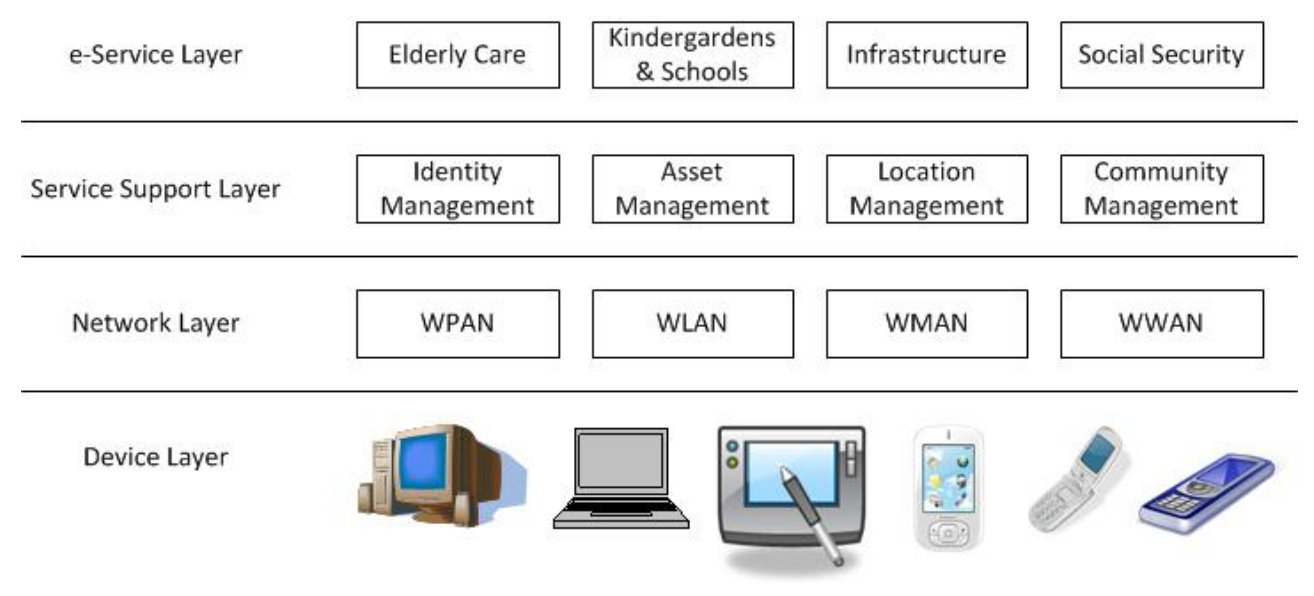

Fig. 3. NIMO Model for Development and Deployment of e-Services

large amount of users guarantees an empiric source to tap from. We will also continue to develop and deploy e-services using the NIMO model to further expand the framework. Some of these will be fully mobile e-services, taking advantage of parameters closely connected to user mobility (e.g. device location), also being adapted for heterogeneous environments and supporting offline work. A presentation of ongoing work on such a service can be found in [14].

\section{ACKNOWLEDGMENT}

This work was supported by the NIMO (Nordic Interaction and Mobility Research Platform) project [15] funded by the EU Interreg IVA North program.

\section{REFERENCES}

[1] ITU, "Key statistical highlights: Itu data release june 2012," June 2012.

[2] N. Al-Dabbous, A. Al-Yatama, and K. Saleh, "Assessment of the trustworthiness of e-service providers," in Proceedings of the 2nd Kuwait Conference on e-Services and e-Systems, KCESS '11, (New York, NY, USA), pp. 24:1-24:7, ACM, 2011.

[3] C.-O. Olsson and A. Rönnbäck, "Collaborative development of public information systems: A case study of "sambruk" e-services development," in eChallenges e-2010 Conference Proceedings, pp. 1-7, 2010.

[4] K. L. Gidlund, "Designing for all and no one - practitioners understandings of citizen driven development of public e-services," in Proceedings of the 12th Participatory Design Conference: Research Papers - Volume 1, PDC '12, (New York, NY, USA), pp. 11-19, ACM, 2012.
[5] H. Gillig and K. Sailer, "User involvement in the innovation process: Development of a framework for e-services," in Proceedings of the 18th International ICE Conference on Engineering, Technology and Innovation (ICE), pp. 1-11, 2012.

[6] T. Grimstad and P. Myrseth, "Information governance as a basis for cross-sector e-services in public administration," in E-Business and EGovernment (ICEE), 2011 International Conference on, pp. 1-4, 2011.

[7] R. Hellman, "Temporal accessibility of e-services," in eChallenges, 2010, pp. 1-8, 2010.

[8] D. Johansson and K. Andersson, "4th generation e-services - requirements for the development of mobile e-services," in eChallenges e-2013 Conference Proceedings, IIMC International Information Management Corporation, 2013.

[9] M. Böcker, H. Huttenrauch, M. Pluke, A. Rodriguez-Ascaso, M. Schneider, and E. Zetterstrom, "Identifying enablers for future e-services," in Pervasive Computing Technologies for Healthcare (PervasiveHealth), 2010 4th International Conference on, pp. 1-5, 2010.

[10] W. L. Ntshinga, "Choreography of intelligent e-services," in Proceedings of the 32nd ACM/IEEE International Conference on Software Engineering - Volume 2, ICSE '10, (New York, NY, USA), pp. 343-344, ACM, 2010.

[11] Skellefteå, “Mitt skellefteå," $2012 . \quad$ [Online]. Available: http://docs.smartstad.se/.

[12] K. Andersson and D. Johansson, "Mobile e-services using html5," in Proceedings of the IEEE 37th Conference on Local Computer Networks Workshops (LCN Workshops), oct 2012.

[13] W3C, "Html 5.1 nightly. a vocabulary and associated apis for html and xhtml," May 2013. [Online]. Available: http://www.w3.org/html/wg/drafts/html/master/.

[14] M. Holmgren, D. Johansson, and K. Andersson, "A web-based time machine with augmented reality," 2013. Submitted for review.

[15] NIMO, "Nordic interaction and mobility research platform," oct 2013. [Online]. Available: http://nimoproject.org/. 